\title{
ON THE SUP-NORM OF MAASS CUSP FORMS OF LARGE LEVEL. III
}

\author{
GERGELY HARCOS AND NICOLAS TEMPLIER
}

\begin{abstract}
Let $f$ be a Hecke-Maass cuspidal newform of square-free level $N$ and Laplacian eigenvalue $\lambda$. It is shown that $\|f\|_{\infty} \ll_{\lambda, \epsilon} N^{-\frac{1}{6}+\epsilon}\|f\|_{2}$ for any $\epsilon>0$.
\end{abstract}

\section{INTRODUCTION}

This note deals with the problem of bounding the sup-norm of eigenfunctions on arithmetic hyperbolic surfaces. It is natural to restrict this problem to HeckeMaass cuspidal newforms which are square-integrable joint eigenfunctions of the Laplacian and Hecke operators. We consider the noncompact modular surface $\Gamma_{0}(N) \backslash \mathfrak{H}$ equipped with its hyperbolic metric and associated measure; the total volume is then asymptotically equal to $N^{1+o(1)}$. We shall $L^{2}$-normalize all HeckeMaass cuspidal newforms $f$ with respect to that measure, namely

$$
\int_{\Gamma_{0}(N) \backslash \mathfrak{H}}|f(z)|^{2} \frac{d x d y}{y^{2}}=1 .
$$

It is interesting to bound the sup-norm $\|f\|_{\infty}$ in terms of the two basic parameters: the Laplacian eigenvalue $\lambda$ and the level $N$.

In the $\lambda$-aspect, the first nontrivial bound is due to Iwaniec and Sarnak [6] who established $\|f\|_{\infty} \ll_{N, \epsilon} \lambda^{\frac{5}{24}+\epsilon}$ for any $\epsilon>0$. Their key idea was to make use of the Hecke operators, through the method of amplification, in order to go beyond $\|f\|_{\infty} \ll_{N} \lambda^{\frac{1}{4}}$ which is valid on any Riemannian surface by [9].

In the $N$-aspect, the "trivial" bound is $\|f\|_{\infty} \ll_{\lambda, \epsilon} N^{\epsilon}$ see [1, 3, 7]. Here and later the dependence on $\lambda$ is continuous. The first nontrivial bound in the $N$ aspect is due to Blomer-Holowinsky [3, p. 673] who proved $\|f\|_{\infty} \ll_{\lambda, \epsilon} N^{-\frac{25}{914}+\epsilon}$, at least for square-free $N$. In [11 the second named author revisited the proof by making a systematic use of geometric arguments, and derived a stronger exponent: $\|f\|_{\infty} \ll_{\lambda, \epsilon} N^{-\frac{1}{22}+\epsilon}$. Helfgott-Ricotta (unpublished) improved some of the estimates in [11] and obtained $\|f\|_{\infty} \ll_{\lambda, \epsilon} N^{-\frac{1}{20}+\epsilon}$. In [5] we introduced a more efficient treatment of the counting problem at the heart of the argument and derived the estimate $\|f\|_{\infty} \ll_{\lambda, \epsilon} N^{-\frac{1}{12}+\epsilon}$. We shall improve this estimate further.

Date: Sep 2011.

2010 Mathematics Subject Classification. 11F12,11D45,14G35.

Key words and phrases. automorphic forms, trace formula, amplification, diophantine approximation.

This work was partially supported by a grant \#209849 from the Simons Foundation (NT) and by EC grant ERG 239277 and OTKA grants K 72731, PD 75126 (GH). 
Theorem 1.1. Let $f$ be an $L^{2}$-normalized Hecke-Maass cuspidal newform of square-free level $N$. Then for any $\epsilon>0$ we have a bound

$$
\|f\|_{\infty} \ll_{\lambda, \epsilon} N^{-\frac{1}{6}+\epsilon}
$$

where the implied constant depends continuously on $\lambda$.

Remarks. (i) It seems that $-\frac{1}{6}$ is the natural exponent for the sup-norm problem in the level aspect. Examples of such exponents are the Weyl exponent $\frac{1}{6}$ (resp. Burgess exponent $\frac{3}{16}$ ) in the subconvexity problem for $\mathrm{GL}_{1}$ in the archimedean (resp. nonarchimedean) aspect, or their doubles in the $\mathrm{GL}_{2^{-}}$ setting.

(ii) Independently, Blomer-Michel [2] obtain a bound of the same quality for Hecke eigenforms on unions of arithmetic ellipsoids. In this paper we are concerned in (2.2) with solutions of an indefinite quadratic equation $\operatorname{det}(\gamma)=$ $l$, whereas arithmetic ellipsoids involve definite quadratic forms.

(iii) From Atkin-Lehner theory we may assume that $\mathfrak{I m} z \gg N^{-1}$ when investigating the sup-norm. The critical range is actually when $\mathfrak{I m} z \leq N^{-\frac{2}{3}+o(1)}$. Otherwise the details of the proof below show that $|f(z)|$ is significantly less than $N^{-\frac{1}{6}}$.

The present note is derived from [10] which is motivated by the comparison of the method in $[6]$ for the $\lambda$-aspect with our method in [5, 11] for the $N$-aspect. The advantage of the new argument in [10] is that it can be adapted to the $\lambda$-aspect to reproduce the bound $\|f\|_{\infty} \ll_{N, \epsilon} \lambda^{\frac{5}{24}+\epsilon}$, which is key for establishing hybrid bounds simultaneously in the $\lambda$ and $N$-aspects. Compared to [5, 11] the reader will find below two improvements coming from a Pell equation and a uniform count of lattice points $[8]$.

\section{Counting Lattice Points}

2.1. Notation. To make this section self-contained we recall the definitions from [5, 11]. Let $\mathrm{GL}_{2}(\mathbb{R})^{+}$act on the upper-half plane $\mathfrak{H}=\{x+i y, y>0\}$ by fractional linear transformations. Denote by $u($,$) the following function of the hyperbolic$ distance:

$$
u(w, z)=\frac{|w-z|^{2}}{4 \mathfrak{I m} w \mathfrak{I m} z} .
$$

For $z \in \mathfrak{H}$ and $l, N \geq 1$ let $M_{*}(z, l, N)$ be the number of matrices $\gamma=\left(\begin{array}{ll}a & b \\ c & d\end{array}\right)$ in $M_{2}(\mathbb{Z})$ such that

$$
\operatorname{det}(\gamma)=l, \quad c \equiv 0(N), \quad u(\gamma z, z) \leq N^{\epsilon}, \quad c \neq 0, \quad(a+d)^{2} \neq 4 l .
$$

We write $f \preccurlyeq g$ meaning that for all $\epsilon>0$ there is a constant $C(\epsilon)>0$ such that $f(N) \leq C(\epsilon) N^{\epsilon} g(N)$ for all $N \geq 1$. To simplify notation we omit the dependence in $\lambda$. For example Theorem 1.1 says $\|f\|_{\infty} \preccurlyeq N^{-\frac{1}{6}}$.

Let $\mathcal{F}(N)$ be the set of $z \in \mathfrak{H}$ such that $\mathfrak{I m} z \geq \mathfrak{I m} \delta z$ for all Atkin-Lehner operators $\delta$ of level $N$. In this section we shall only use the fact ([5, Lemma 2.2]) that for all $z=x+i y \in \mathcal{F}(N)$, we have $N y \gg 1$ and that for all $(a, b) \in \mathbb{Z}^{2}$ 
distinct from $(0,0)$ we have

$$
|a z+b|^{2} \geq \frac{1}{N}
$$

2.2. Lattice points. We have the following uniform estimate for the number of lattice points in a disc ([8, Lemma 2]):

Lemma 2.1. Let $M$ be a euclidean lattice of rank 2 and $D$ be a disc of radius $R>0$ in $M \otimes_{\mathbb{Z}} \mathbb{R}$ (not necessarily centered at 0 ). If $\lambda_{1} \leq \lambda_{2}$ are the successive minima of $M$, then

$$
\# M \cap D \ll 1+\frac{R}{\lambda_{1}}+\frac{R^{2}}{\lambda_{1} \lambda_{2}}
$$

Remarks. (i) Let $d(M)>0$ be the covolume of $M$. Minkowski's second Theorem asserts that $\lambda_{1} \lambda_{2} \asymp d(M)$. When $R \rightarrow \infty$, the leading term of (2.4) is $\frac{R^{2}}{d(M)}$ as expected.

(ii) It is easier to establish the upper-bound $\ll 1+\frac{R^{2}}{\lambda_{1}^{2}}$ (which also has the advantage of having only two terms). One can verify that

$$
1+\frac{R}{\lambda_{1}}+\frac{R^{2}}{\lambda_{1} \lambda_{2}} \ll 1+\frac{R^{2}}{\lambda_{1}^{2}} .
$$

Thus the estimate in (2.4) is always better.

(iii) We have equality (up to a constant) in (2.5) if and only if $R \ll \lambda_{1}$. In the applications below it is often the case that $R \ll \lambda_{1}$. However this is not always the case, and then the improvement of $(2.4)$ on the easier bound is significant.

2.3. Counting. The following is an improvement on [5, Lemma 4.2]:

Lemma 2.2. Let $z=x+i y \in \mathcal{F}(N)$ and $1 \leq L \leq N^{O(1)}$. Then

$$
\sum_{1 \leq l \leq L} M_{*}(z, l, N) \preccurlyeq \frac{L}{N y}+\frac{L^{\frac{3}{2}}}{N^{\frac{1}{2}}}+\frac{L^{2}}{N} .
$$

If we restrict to $l$ being a perfect square, then one can improve by a factor $L^{\frac{1}{2}}$ :

$$
\sum_{\substack{1 \leq l \leq L, l \text { is a square }}} M_{*}(z, l, N) \preccurlyeq \frac{L^{\frac{1}{2}}}{N y}+\frac{L}{N^{\frac{1}{2}}}+\frac{L^{\frac{3}{2}}}{N} .
$$

Proof. We briefly recall the beginning of the argument in [5, Lemma 4.2]. Let $\gamma=\left(\begin{array}{ll}a & b \\ c & d\end{array}\right)$ satisfy 2.2 . In coordinates we have

$$
\left|-c z^{2}+(a-d) z+b\right|^{2} \leq L y^{2} N^{\epsilon}
$$

As in $\mid 5,6]$ we verify that $|c| \preccurlyeq L^{\frac{1}{2}} / y$, so there are $\preccurlyeq L^{\frac{1}{2}} /(N y)$ possible values of $c$.

Consider the lattice $\langle 1, z\rangle$ inside $\mathbb{C}$. Its covolume equals $y$ and its shortest length is at least $N^{-1 / 2}$ by $(2.3)$. In the inequality $(2.8)$ we are counting lattice points 
$(a-d, b)$ in a disc of volume $\preccurlyeq L y^{2}$ centered at $c z^{2}$. Hence by Lemma (2.1), there are $\preccurlyeq 1+\frac{L^{\frac{1}{2}} y}{N^{-\frac{1}{2}}}+\frac{L y^{2}}{y}$ possible pairs $(a-d, b)$ for each value of $c$.

As in $\mid 5,6]$ one can deduce from (2.8) that $|a+d| \preccurlyeq L^{\frac{1}{2}}$. This concludes the proof of (2.6).

For (2.7) we instead use the identity

$$
(a-d)^{2}+4 b c=(a+d)^{2}-4 l .
$$

The left-hand side is non-zero by assumption (2.2). Since $l$ is a perfect square, for each given triple $(a-d, b, c)$ the number of pairs $(a+d, l)$ satisfying $(2.9)$ is $\preccurlyeq 1$. This concludes the proof.

The following is a refinement of (2.7).

Lemma 2.3. Let $z=x+i y \in \mathcal{F}(N)$ and $1 \leq l_{1} \leq \Lambda \leq N^{O(1)}$. Then

$$
\sum_{1 \leq l_{2} \leq \Lambda} M_{*}\left(z, l_{1} l_{2}^{2}, N\right) \preccurlyeq \frac{\Lambda^{\frac{3}{2}}}{N y}+\frac{\Lambda^{3}}{N^{\frac{1}{2}}}+\frac{\Lambda^{\frac{9}{2}}}{N} .
$$

Proof. Let $\gamma=\left(\begin{array}{ll}a & b \\ c & d\end{array}\right)$ satisfy 2.2 . We have

$$
\left|-c z^{2}+(a-d) z+b\right|^{2} \leq l_{1} l_{2}^{2} y^{2} N^{\epsilon} .
$$

This implies $|c| \preccurlyeq \Lambda^{\frac{3}{2}} / y$, so there are $\preccurlyeq \Lambda^{\frac{3}{2}} /(N y)$ possible values of $c$.

For each value of $c$, we again apply Lemma 2.1 to the lattice $\langle 1, z\rangle$ of covolume $y$ and shortest length at least $N^{-\frac{1}{2}}$. In the inequality (2.11) we are counting lattice points $(a-d, b)$ in a disc of volume $\preccurlyeq \Lambda^{3} y^{2}$. This implies that there are $\preccurlyeq 1+\frac{\Lambda^{\frac{3}{2}} y}{N^{-\frac{1}{2}}}+\frac{\Lambda^{3} y^{2}}{y}$ possible pairs $(a-d, b)$ satisfying (2.11).

Further, since $\operatorname{det}(\gamma)=l_{1} l_{2}^{2}$, we have:

$$
(a-d)^{2}+4 b c=(a+d)^{2}-4 l_{1} l_{2}^{2}
$$

The left-hand side is already determined by the values of $c$ and $(a-d, b)$. It is nonzero by assumption 2.2 . This is a generalized Pell equation in the remaining variables $a+d$ and $l_{2}$.

Without loss of generality we can assume that $l_{1}$ is square-free. One can deduce from $(2.8)$ that $|a+d| \preccurlyeq \Lambda^{\frac{3}{2}}$. If $l_{1}=1$ then we are done with a divisor bound as in the proof of 2.7 .

If $l_{1}>1$ then we write the solutions of the equation in terms of the fundamental unit. The fundamental unit is always greater than $\frac{1+\sqrt{5}}{2}=1.618 \cdots$, which is bounded away from 1 (for better estimates, see [4] and the references herein). We deduce that the number of pairs $\left(a+d, l_{2}\right)$ of solutions of $(2.12)$ is $\ll \Lambda^{o(1)} \preccurlyeq 1$.

The total number of $\gamma$ 's is

$$
\preccurlyeq \frac{\Lambda^{\frac{3}{2}}}{N y} \cdot\left(1+\Lambda^{\frac{3}{2}} N^{\frac{1}{2}} y+\Lambda^{3} y\right) .
$$

This concludes the proof of the lemma. 
2.4. Special matrices. We let $M_{u}(z, l, N)$ be the number of matrices satisfying (2.2) but with the condition $c=0$ instead of $c \neq 0$ (upper-triangular).

Lemma 2.4. Let $z=x+i y \in \mathcal{F}(N)$ and $1 \leq \Lambda \leq N^{O(1)}$. Then the following estimates hold, where $l_{1}, l_{2}$ run through prime numbers:

$$
\sum_{1 \leq l_{1}, l_{2} \leq \Lambda} M_{u}\left(z, l_{1} l_{2}, N\right) \preccurlyeq \Lambda+\Lambda^{2} N^{\frac{1}{2}} y+\Lambda^{3} y,
$$

$$
\sum_{1 \leq l_{1}, l_{2} \leq \Lambda} M_{u}\left(z, l_{1} l_{2}^{2}, N\right) \preccurlyeq \Lambda+\Lambda^{\frac{5}{2}} N^{\frac{1}{2}} y+\Lambda^{4} y
$$

$$
\sum_{1 \leq l_{1}, l_{2} \leq \Lambda} M_{u}\left(z, l_{1}^{2} l_{2}^{2}, N\right) \preccurlyeq 1+\Lambda^{2} N^{\frac{1}{2}} y+\Lambda^{4} y .
$$

Proof. We need to count the number of matrices $\gamma=\left(\begin{array}{ll}a & b \\ 0 & d\end{array}\right) \in M_{2}(\mathbb{Z})$ such that

$$
|(a-d) z+b|^{2} \leq a d y^{2} N^{\epsilon}
$$

and $a d=l_{1} l_{2}\left(\right.$ resp. $a d=l_{1} l_{2}^{2}$, and $\left.a d=l_{1}^{2} l_{2}^{2}\right)$.

We again consider the lattice $\langle 1, z\rangle$ of covolume $y$ and shortest length at least $N^{-\frac{1}{2}}$. In the inequality 2.17$)$ we are counting lattice points $(a-d, b)$ in a disc of volume $\preccurlyeq a d y^{2}$.

We consider (2.14) first. There are $\preccurlyeq 1+\Lambda N^{\frac{1}{2}} y+\Lambda^{2} y$ possible pairs of integers $(a-d, b)$ satisfying (2.17). Each pair gives rise to $O(\Lambda)$ matrices $\gamma$ (this is because $\left.a d=l_{1} l_{2}\right)$.

Next we consider $(2.15)$. There are $\preccurlyeq 1+\Lambda^{\frac{3}{2}} N^{\frac{1}{2}} y+\Lambda^{3} y$ pairs of integers $(a-d, b)$ satisfying (2.17). Each pair gives rise to $O(\Lambda)$ matrices $\gamma$ (this is because $\left.a d=l_{1} l_{2}^{2}\right)$.

Finally we consider (2.16). There are $\preccurlyeq 1+\Lambda^{2} N^{\frac{1}{2}} y+\Lambda^{4} y$ pairs of integers $(a-d, b)$ satisfying (2.17). Since $l_{1}$ and $l_{2}$ are primes, we have either $(a=1, d=$ $\left.l_{1}^{2} l_{2}^{2}\right)$ or $\left(a=l_{1}, d=l_{1} l_{2}^{2}\right)$ or $\left(a=l_{1}^{2}, d=l_{2}^{2}\right)$, or equivalent configurations. In each configuration, and for a given value of $a-d$, there are $\Lambda^{o(1)}$ pairs $(a, d)$. Thus each pair $(a-d, b)$ gives rise to $\Lambda^{o(1)}$ matrices $\gamma$.

We note that a similar proof also yields:

$$
\sum_{\substack{1 \leq l \leq L \\ l \text { prime }}} M_{u}(z, l, N) \preccurlyeq 1+L^{\frac{1}{2}} N^{\frac{1}{2}} y+L y .
$$

Finally let $M_{p}(z, l, N)$ be the number of matrices satisfying (2.2) but instead with the condition $(a+d)^{2}=4 l$ (parabolic) and with no restriction on $c \equiv 0(N)$. Then [5, Lemma 4.1] gives

$$
M_{p}(z, l, N)=2 \delta_{\square}(l), \quad 1 \leq l<y^{-2} N^{-\epsilon} .
$$

Here $\delta_{\square}(l)=1,0$ depending on whether $l$ is a perfect square or not.

We let $M(z, l, N):=M_{*}(z, l, N)+M_{u}(z, l, N)+M_{p}(z, l, N)$ which is the number of matrices satisfying the first three conditions in (2.2). 


\section{Proof of Theorem 1.1}

Applying the amplification method of Friedlander-Iwaniec as in [6] and [5, §3], we have

$$
\Lambda^{2}|f(z)|^{2} \preccurlyeq \sum_{l \geq 1} \frac{y_{l}}{\sqrt{l}} M(z, l, N)
$$

Here $\Lambda^{2}>0$ is the amplifier length and the sequence $y_{l} \in \mathbb{R}_{\geq 0}$ satisfies:

$$
y_{l}:= \begin{cases}\Lambda, & l=1, \\ 1, & l=l_{1} \text { or } l_{1} l_{2} \text { or } l_{1} l_{2}^{2} \text { or } l_{1}^{2} l_{2}^{2} \text { with } \Lambda<l_{1}, l_{2}<2 \Lambda \text { primes } \\ 0, & \text { otherwise. }\end{cases}
$$

By [11, $\S 3.2],|f(x+i y)| \preccurlyeq(N y)^{-\frac{1}{2}}$. Thus we may assume that $y<N^{-\frac{2}{3}}$ when establishing Theorem 1.1. Without loss of generality we can also assume $z=x+i y \in \mathcal{F}(N)$.

We shall choose $\Lambda=N^{\frac{1}{3}-\frac{\epsilon}{4}}$. This implies $\Lambda^{4}<y^{-2} N^{-\epsilon}$, thus the condition in (2.19) is satisfied. Therefore the contribution in (3.1) of the parabolic matrices is $\ll \Lambda$ using $(2.19)$.

The contribution in (3.1) of the upper-triangular matrices with $l=1$ is $\preccurlyeq \Lambda\left(1+N^{\frac{1}{2}} y+y\right)$ using (2.18). For $\Lambda<l<2 \Lambda$ it is $\preccurlyeq \Lambda^{-\frac{1}{2}}+N^{\frac{1}{2}} y+\Lambda^{\frac{1}{2}} y$ using (2.18) again. For $\Lambda^{2}<l<4 \Lambda^{2}$ it is $\preccurlyeq 1+\Lambda N^{\frac{1}{2}} y+\Lambda^{2} y$ using (2.14) of Lemma 2.4. For $\Lambda^{3}<l<8 \Lambda^{3}$ it is $\preccurlyeq \Lambda N^{\frac{1}{2}} y+\Lambda^{\frac{5}{2}} y$ using (2.15) of Lemma 2.4. For $l>\Lambda^{4}$ it is $\preccurlyeq N^{\frac{1}{2}} y+\Lambda^{2} y$ using $(2.16)$ of Lemma 2.4 .

It now remains to consider the matrices in 2.2 counted by $M_{*}$. The contribution in (3.1) of $l=1$ is $\preccurlyeq \Lambda\left(\frac{1}{N y}+N^{-\frac{1}{2}}\right)$ using (2.6) in Lemma 2.2. For $\Lambda<l<2 \Lambda$ it is $\preccurlyeq \frac{\Lambda^{\frac{1}{2}}}{N y}+\frac{\Lambda}{N^{\frac{1}{2}}}+\frac{\Lambda^{\frac{3}{2}}}{N}$ using 2.6 again. For $\Lambda^{2}<l<4 \Lambda^{2}$ it is $\preccurlyeq \frac{\Lambda}{N y}+\frac{\Lambda^{2}}{N^{\frac{1}{2}}}+\frac{\Lambda^{3}}{N}$ using 2.6 again. For $\Lambda^{3}<l<8 \Lambda^{3}$ it is $\preccurlyeq \frac{\Lambda}{N y}+\frac{\Lambda^{\frac{5}{2}}}{N^{\frac{1}{2}}}+\frac{\Lambda^{4}}{N}$ using Lemma 2.3. For $l>\Lambda^{4}$ it is $\preccurlyeq \frac{1}{N y}+\frac{\Lambda^{2}}{N^{\frac{1}{2}}}+\frac{\Lambda^{4}}{N}$ using (2.7).

Altogether we obtain that

$$
\Lambda^{2}|f(z)|^{2} \preccurlyeq \Lambda+\frac{\Lambda^{\frac{5}{2}}}{N^{\frac{1}{2}}}+\frac{\Lambda^{4}}{N} .
$$

Choosing $\Lambda:=N^{\frac{1}{3}-\frac{\epsilon}{4}}$, all three terms above are equal to $N^{\frac{1}{3}+o(1)}$. This concludes the proof of Theorem 1.1 .

Remark. The conclusion of Theorem 1.1 holds true for Hecke-Maass cuspidal newforms of an arbitrary nebentypus and also for holomorphic modular forms. Indeed the amplification method again yields the inequality (3.1) above and the rest of the proof goes through without change. Also the assumption that $f$ be a newform is not necessary since Atkin-Lehner theory reduces the general case to the case of newforms. For an oldform $f$, the bound would be in terms of the level from which $f$ was induced.

Acknowledgements. We thank the referee for his helpful comments. 


\section{REFERENCES}

[1] A. Abbes and E. Ullmo, Comparaison des métriques d'A rakelov et de Poincaré sur $X_{0}(N)$, Duke Math. J. 80 (1995), no. 2, 295-307.

[2] V. Blomer and Ph. Michel, Hybrid bounds for automorphic forms on ellipsoids over number fields, available at http://arxiv.org/abs/1110.4526.

[3] V. Blomer and R. Holowinsky, Bounding sup-norms of cusp forms of large level, Invent. Math. 179 (2010), no. 3, 645-681.

[4] E. Fouvry and F. Jouve, A positive density of fundamental discriminants with large regulator, available at http://www .math.u-psud.fr/ fouvry/.

[5] G. Harcos and N. Templier, On the sup-norm of Maass cusp forms of large level. II, Int. Math. Res. Not. (2011), Art. ID rnr202, 11pp.

[6] H. Iwaniec and P. Sarnak, $L^{\infty}$ norms of eigenfunctions of arithmetic surfaces, Ann. of Math. (2) 141 (1995), no. 2, 301-320.

[7] Ph. Michel and E. Ullmo, Points de petite hauteur sur les courbes modulaires $X_{0}(N)$, Invent. Math. 131 (1998), no. 3, 645-674.

[8] W. M. Schmidt, Asymptotic formulae for point lattices of bounded determinant and subspaces of bounded height, Duke Math. J. 35 (1968), 327-339.

[9] A. Seeger and C. D. Sogge, Bounds for eigenfunctions of differential operators, Indiana Univ. Math. J. 38 (1989), no. 3, 669-682.

[10] N. Templier, Hybrid sup-norm bounds for Hecke-Maass cusp forms, Submitted.

[11] _ On the sup-norm of Maass cusp forms of large level, Selecta Math. (N.S.) 16 (2010), no. 3, 501-531.

Alfréd Rényi Institute of Mathematics, Hungarian Academy of Sciences, POB 127, Budapest H-1364, Hungary

E-mail address: gharcos@renyi.hu

Department of Mathematics, Fine Hall, Washington Road, Princeton, NJ 085441000 .

E-mail address: templier@math.princeton.edu 